\title{
AIP Aponed phosics

\section{Discussion on device structures and hermetic encapsulation for SiOx random access memory operation in air}

Fei Zhou, Yao-Feng Chang, Yanzhen Wang, Yen-Ting Chen, Fei Xue, Burt W. Fowler, and Jack C. Lee

Citation: Applied Physics Letters 105, 163506 (2014); doi: 10.1063/1.4900422

View online: http://dx.doi.org/10.1063/1.4900422

View Table of Contents: http://scitation.aip.org/content/aip/journal/apl/105/16?ver=pdfcov

Published by the AIP Publishing

\section{Articles you may be interested in}

Effect of plasma treatment of resistive layer on a $\mathrm{Cu} / \mathrm{SiOx} / \mathrm{Pt}$ memory device

J. Vac. Sci. Technol. A 32, 02B111 (2014); 10.1116/1.4859235

Mechanism of power consumption inhibitive multi-layer $\mathrm{Zn}: \mathrm{SiO} 2 / \mathrm{SiO} 2$ structure resistance random access memory

J. Appl. Phys. 114, 234501 (2013); 10.1063/1.4843695

Insertion of a Si layer to reduce operation current for resistive random access memory applications

Appl. Phys. Lett. 102, 252902 (2013); 10.1063/1.4812304

Hopping conduction distance dependent activation energy characteristics of $\mathrm{Zn}$ :SiO2 resistance random access memory devices

Appl. Phys. Lett. 102, 133503 (2013); 10.1063/1.4799655

Guided filament formation in NiO-resistive random access memory by embedding gold nanoparticles Appl. Phys. Lett. 100, 083105 (2012); 10.1063/1.3688053

Frustrated by

old technology?

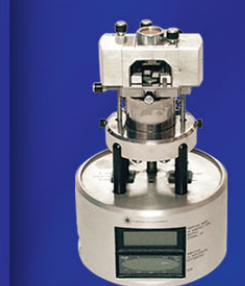

Is your AFM dead

and can't be repaired?

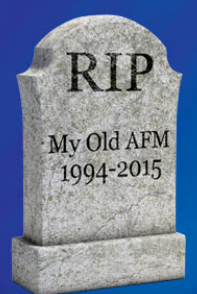

Sick of bad customer support?

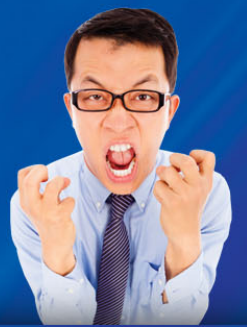

It is time to upgrade your AFM

Minimum $\$ 20,000$ trade-in discount for purchases before August 31st

Asylum Research is today's technology leader in AFM 


\title{
Discussion on device structures and hermetic encapsulation for $\mathrm{SiO}_{x}$ random access memory operation in air
}

\author{
Fei Zhou, ${ }^{1}$ Yao-Feng Chang, ${ }^{1, a)}$ Yanzhen Wang, ${ }^{1}$ Yen-Ting Chen, ${ }^{1}$ Fei Xue, ${ }^{1}$ \\ Burt W. Fowler, ${ }^{2}$ and Jack C. Lee ${ }^{1}$ \\ ${ }^{1}$ Microelectronics Research Center, Department of Electrical and Computer Engineering, \\ The University of Texas at Austin, Austin, Texas 78758, USA \\ ${ }^{2}$ PrivaTran, LLC, 1250 Capital of Texas Highway South, Bldg 3, Ste 400, Austin Texas 78746, USA
}

(Received 9 September 2014; accepted 12 October 2014; published online 21 October 2014)

\begin{abstract}
An edge-free structure and hermetic encapsulation technique are presented that enable $\mathrm{SiO}_{\mathrm{x}}$-based resistive random-access memory (RRAM) operation in air. A controlled etch study indicates that the switching filament is close to the $\mathrm{SiO}_{\mathrm{x}}$ surface in devices with an exposed $\mathrm{SiO}_{\mathrm{x}}$ edge. Electrical test of encapsulated, edge-free devices in 1 atmosphere air indicates stable switching characteristics, unlike devices with an edge. This work demonstrates that $\mathrm{SiO}_{\mathrm{x}} \mathrm{RRAM}$ is able to operate in air with proper encapsulation and an edge-free structure. The resistive switching failure mechanism when operating in air is explained by the oxidation of hydrogen-complexed defects in the switching filament. (C 2014 AIP Publishing LLC. [http://dx.doi.org/10.1063/1.4900422]
\end{abstract}

The ever-increasing demand for higher memory density and computing energy efficiency requires future nonvolatile random access memory (RAM) devices to have low cost, ultimate scaling potential, high speed, and good data retention. ${ }^{1,2}$ As a promising candidate for such applications, amorphous silicon oxide $\left(\mathrm{SiO}_{\mathrm{x}}\right)$ memristive materials have been intensively studied. ${ }^{3-5}$ The $\mathrm{Si} / \mathrm{SiO}_{2}$ system has been utilized extensively in the semiconductor industry for over half a century. All advanced fabrication technologies evolved around this materials system, which gives it unparalleled advantages in terms of device miniaturization and scaling efficiency. Recent reports indicate that $\mathrm{SiO}_{\mathrm{x}}$ resistive random access memory (RRAM) has good retention in air ambient and sub- 100 ns pulsed switching capability, ${ }^{6-8}$ but resistive state switching must be performed a in vacuum environment below $10^{-3}$ mbars or an oxygen-free ambient. This impedes implementation of $\mathrm{SiO}_{\mathrm{x}}$-based RRAM devices in complementary metal-oxide-semiconductor (CMOS) technology, and the question of whether $\mathrm{SiO}_{\mathrm{x}}$-based RRAM devices can operate in air ambient becomes a critical factor limiting its application. Only very recently, a study on Si-rich silica as resistive switching material has demonstrated resistive state switching in air; ${ }^{9}$ however, the device was operated primarily in bipolar mode. The differences in switching characteristics and charge transport suggest that the fundamental operating mechanisms may be different in Si-rich silica as compared with $\mathrm{SiO}_{\mathrm{x}}$ materials that exhibit unipolar properties in vacuum ambient. ${ }^{3}$ More studies on the operation of $\mathrm{SiO}_{\mathrm{x}}$-based RRAM devices in air are needed, particularly their electroforming process, reversible switching characteristics, and switching mechanisms. In this paper, we fabricated devices with two different structures, namely, those with a continuous $\mathrm{SiO}_{\mathrm{x}}$ layer and previously reported structures with an etched $\mathrm{SiO}_{\mathrm{x}}$ layer and an exposed edge. Both devices were first electroformed in vacuum and then tested in air without any encapsulation. An encapsulation process

\footnotetext{
${ }^{\text {a) }}$ Author to whom correspondence should be addressed. Electronic mail: yfchang@utexas.edu
}

using epoxy-based negative-tone photoresist (SU-8) photoresist was then performed on both devices, followed by electrical measurement in air. To further understand the reason why un-encapsulated devices fail to switch in air, we performed a controlled etch experiment to locate the active switching element. Lastly, we provide a potential explanation for the failure of resistive switching in air that involves oxygen reactions with hydrogen-complexed defects in $\mathrm{SiO}_{\mathrm{x}}$.

Heavily doped n-type (100) silicon wafers with resistivity of $10^{-3} \Omega \mathrm{cm}$ were used as substrate for $\mathrm{SiO}_{\mathrm{x}} \mathrm{RRAM}$ device fabrication. Native oxide was first removed using buffered oxide etch (BOE). Then a layer of $\mathrm{SiO}_{x}$ with a thickness of $51 \mathrm{~nm}$ was deposited using electron-beam evaporation at $130{ }^{\circ} \mathrm{C}$. The measured refractive index was 1.46 at $550 \mathrm{~nm}$. For devices formed in a continuous $\mathrm{SiO}_{\mathrm{x}}$ layer without an exposed $\mathrm{SiO}_{\mathrm{x}}$ edge (referred to as "bulk devices"), photoresist (AZ 5214) was patterned for lift-off purposes. A $250 \mathrm{~nm}$-thick tantalum nitride (TaN) layer was deposited using reactive sputtering and lifted-off to form the top electrode. Finally, the samples were cleaned with isopropyl alcohol, water, and nitrogen blow dry. In the case of device structures with a $\mathrm{SiO}_{\mathrm{x}}$ edge (referred to as "edge devices"), a $250 \mathrm{~nm}$-thick TaN layer was deposited using reactive sputtering onto the $51 \mathrm{~nm}$-thick $\mathrm{SiO}_{\mathrm{x}}$. The TaN layer was then patterned and dry-etched using $\mathrm{CF}_{4}$ to form the top electrode. The $\mathrm{SiO}_{\mathrm{x}}$ in the field region was then removed with $\mathrm{BOE}$, leaving RRAM devices with an etched $\mathrm{SiO}_{\mathrm{x}}$ edge between top TaN and bottom silicon electrodes.

After electroforming in vacuum, we then used hard-baked SU-8 resist to encapsulate the devices. The fabricated RRAM devices require the encapsulation layer to have good film uniformity over three-dimensional topography. The spun-on SU-8 photoresist meets this requirement and has been used in various circumstances for hermetic sealing purposes. ${ }^{10}$ With proper planarization to reduce topography, other common materials (e.g., silicon nitride silicon oxynitride) may serve the same hermetic encapsulation role. ${ }^{21,22}$ The SU-8 2002 photoresist ( $\sim 2 \mu \mathrm{m}$ thick) was applied to the sample to ensure full coverage of the top surface, followed by prebake at $95^{\circ} \mathrm{C}$ 
for $2 \mathrm{~min}$. The sample then underwent an ultraviolet (i-line) flood exposure for $10 \mathrm{~s}$. The post-exposure bake was performed at $95^{\circ} \mathrm{C}$ for $2 \mathrm{~min}$ on a hotplate. After the sample cooled down to room temperature, it was hard-baked at $120^{\circ} \mathrm{C}$ for $8 \mathrm{~min}$ in an air oven so that the SU-8 epoxy became highly cross-linked. The encapsulation process was the same for both bulk devices and edge devices. A profilometer measured the final encapsulation layer to be about $1 \mu \mathrm{m}$ thick. The adhesion between SU-8 layer and the $\mathrm{SiO}_{\mathrm{x}}$ RRAM device is very strong, where even heated acetone with ultrasonic agitation cannot remove the SU-8 layer from the sample. To achieve the best hermetic seal, the photoresist layer was not patterned so that the SU-8 epoxy covered the entire top surface of the samples. A tungsten probe tip was used to penetrate the photoresist and perform electrical measurements.

Figures 1(a) and 1(b) demonstrate the resistive switching characteristics of both bulk device and edge device without encapsulation. Typical vacuum electroforming processes for both devices are shown in the insets of Figure 1. A forward/reverse voltage sweep from 0 to $18 \mathrm{~V}$ is applied to electroform the edge device. During the reverse sweep, the current initially fluctuated near the $10^{-6} \mathrm{~A}$ level, then increased to $10^{-3} \mathrm{~A}$, indicating the completion of the electroforming process. As for the bulk device, a forward/reverse sweep to a much larger voltage $(35 \mathrm{~V})$ was used. During the reverse sweep, a sharp current increase to $10 \mathrm{nA}$ was observed at $\sim 30 \mathrm{~V}$, which marked the beginning of the electroforming process. ${ }^{11}$ During the reverse sweep, the current fluctuated near $10^{-6} \mathrm{~A}$ and then increased to $10^{-3} \mathrm{~A}$, similar to the behavior of the edge device. After electroforming, several 0 to $8 \mathrm{~V}$ sweeps were performed in vacuum on both samples to observe the resistive state switching characteristics (i.e., the "on state" and "off state" curves). It can be seen that the Set voltage is $3 \mathrm{~V}$ for both bulk and edge devices. The Reset voltage is about $4-5 \mathrm{~V}$ for the edge device, and $5-6 \mathrm{~V}$ for the bulk device. Note that Set voltage is the voltage needed to switch the device from off-state (i.e., high resistance state, HRS, and Off) to on-state (i.e., low resistance state, LRS, and On); and Reset voltage is the voltage needed to switch from LRS to HRS. The On/Off ratio is about $10^{6}$ for the edge device, and $10^{3}$ for the bulk device.

We suggest that the difference in electroforming and switching behaviors for the two device types originates from structural influences on the electroforming process. ${ }^{12}$ During electroforming, the $\mathrm{SiO}_{\mathrm{x}}$ edge provides an effective pathway for oxygen atoms to migrate out of the $\mathrm{SiO}_{\mathrm{x}}$ layer, thus forming a Si-rich filament that is electrically active and supports resistive switching. For bulk devices, oxygen removal during electroforming may be less effective than in edge devices due to lack of edge and a longer migration distance. Even when oxygen diffusion is enhanced by the use of highly porous, e-beam evaporated $\mathrm{SiO}_{\mathrm{x}}$ as the switching layer, bulk devices still have significantly larger electroforming voltage, presumably due to the greater difficulty in removing oxygen atoms from bulk regions within the $\mathrm{SiO}_{\mathrm{x}}$ layer. ${ }^{13}$ This difficulty to remove oxygen from bulk $\mathrm{SiO}_{\mathrm{x}}$ layers may also affect the composition of the Si-rich filament, resulting in increased internal filament resistance and therefore larger reset voltages in bulk devices.

To confirm the necessity of encapsulation, edge devices without encapsulation were first electroformed in vacuum, then tested in a controlled ambient with varying oxygen pressure using the same configuration and equipment. In Figure 2(a), voltage sweeps from 0 to $8 \mathrm{~V}$ were used to switch edge devices from HRS to LRS and back to HRS. The results show that the edge device exhibits progressively less resistive switching behavior as oxygen pressure increases, with the state transitions occurring closer to each other as pressure increases until switching is no longer observed at 100 Torr. Figure 2(b) shows that the peak current level at a read voltage of $4 \mathrm{~V}$ decreases significantly as oxygen pressure increases, which means that the device gradually loses its ability to switch from HRS to LRS. It is believed that oxygen oxidizes the Si-rich filament so that the device loses its switching capability and memory characteristics. Thus, an encapsulation layer that prevents oxygen from diffusing into the device and reacting with the Si-rich filament is required for $\mathrm{SiO}_{\mathrm{x}}$ RRAM to operate in air ambient. More detailed experimental results and discussions can be found in our previous report on oxygen-induced bimodal failure phenomenon in $\mathrm{SiO}_{\mathrm{x}}$-based RRAM. ${ }^{13}$

To further understand the Si-rich filament's reaction with air ambient, we performed controlled etch studies to identify its physical location. A set of both edge and bulk devices were first electroformed and operated over several switching cycles. Some devices were programed to the LRS
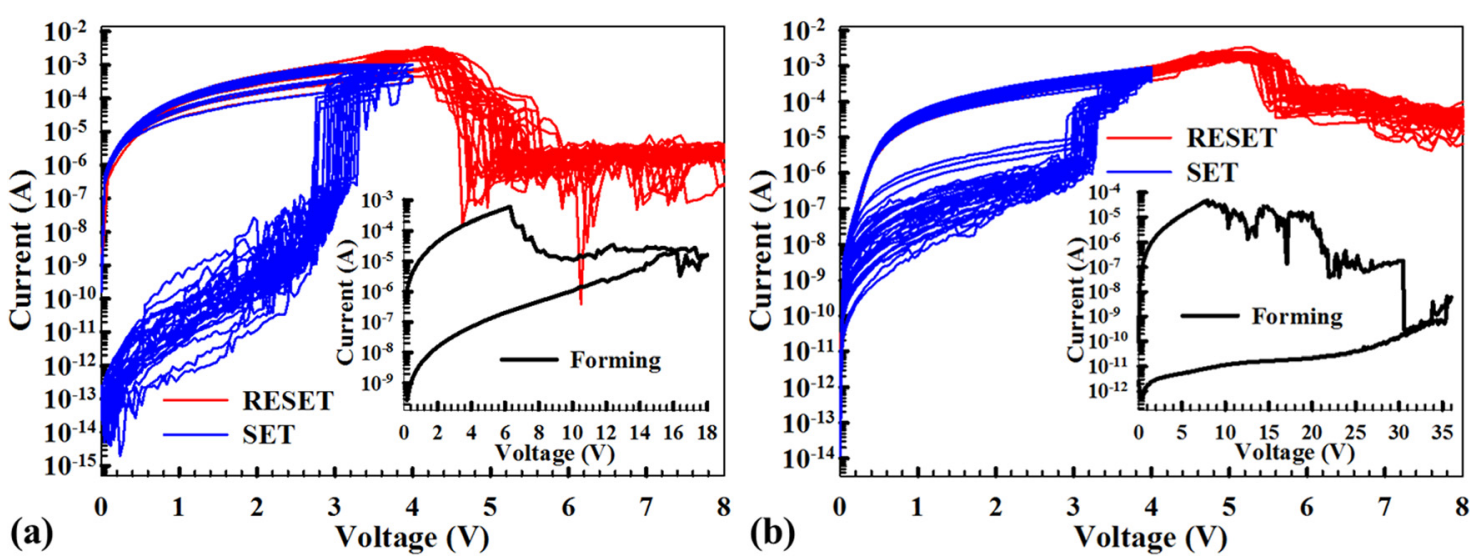

FIG. 1. (a) Resistive switching behavior of $\mathrm{SiO}_{\mathrm{x}}$ RRAM with an edge structure. Inset shows the electroforming process. (b) Resistive switching behavior of $\mathrm{SiO}_{\mathrm{x}}$ RRAM with a bulk structure. Inset shows the electroforming process. 

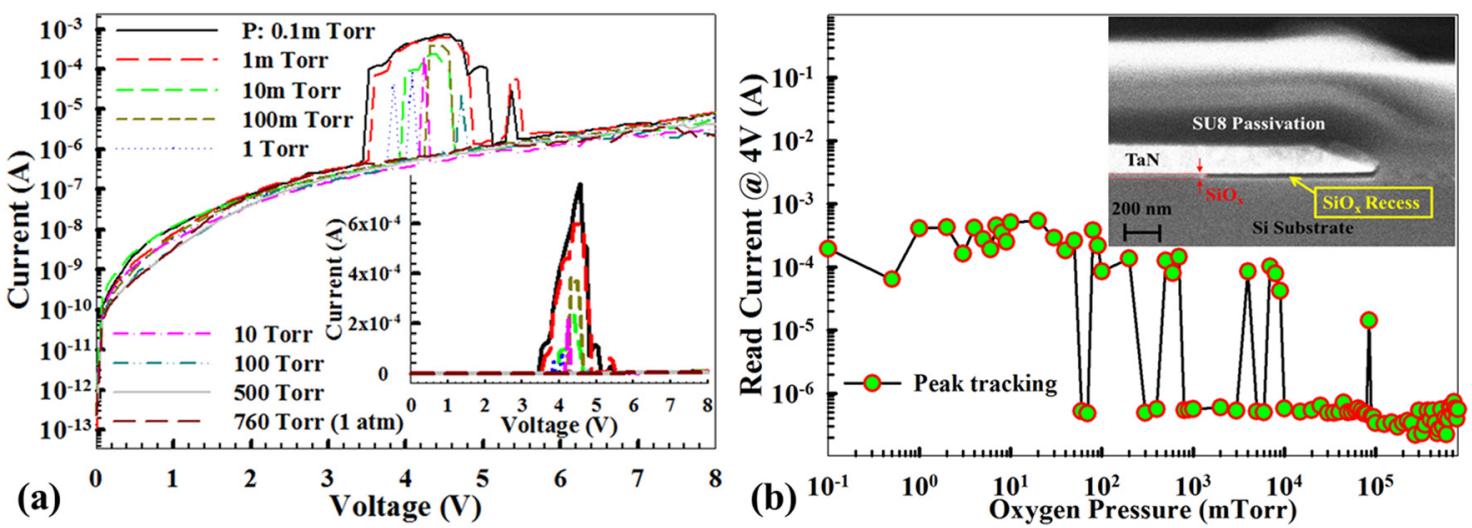

FIG. 2. (a) Resistive switching of edge device measured under varying oxygen pressure. The inset is the same data plotted using a linear scale. (b) Peak current measured in Figure 2(a) at varying oxygen pressure. The inset shows cross-section SEM image of edge device with SU-8 encapsulation.

and some to the HRS. Device $\mathrm{SiO}_{2}$ layers were etched in dilute hydrogen fluoride $\left(1: 100 \mathrm{HF}: \mathrm{H}_{2} \mathrm{O}\right)$ at a rate of $\sim 0.2 \mathrm{~nm} / \mathrm{s}$ for various etch times and device state was measured after each etching step. A device state change indicated that the Si-rich filament was attacked by the etchant, leading to an estimate of the lateral depth into the $\mathrm{SiO}_{2}$ layer where the filament was located. A total of 176 edge devices and 66 bulk devices were included in the investigation and the experiment was repeated three times in order to validate the results. For bulk devices, Figure 3 shows that most filaments are located more than $10 \mathrm{~nm}$ from the electrode edge since only $10 \%$ of devices were attacked by the etch at a depth of $5 \mathrm{~nm}$. This result supports our hypothesis that oxygen must travel longer distances to reach the surface in order to electroform the bulk device, thus resulting in a Si-rich filament located farther from the electrode edge. In contrast, for edge devices, Figure 3 shows that all filaments are within $10 \mathrm{~nm}$ of the exposed edge with $\sim 50 \%$ located within $\sim 4 \mathrm{~nm}$ of the original edge surface. The controlled etch study confirms that the Si-rich filament in edge devices is located very near the edge and, as a result, may readily react with oxygen in air ambient. As discussed further below, this oxidation severely impairs state switching.

Encapsulated bulk and edge devices were then tested in air to determine their resistive switching (RS) characteristics. Only encapsulated bulk devices were able to switch in air. While the presence of $\mathrm{SiO}_{\mathrm{x}}$ edge has often been considered

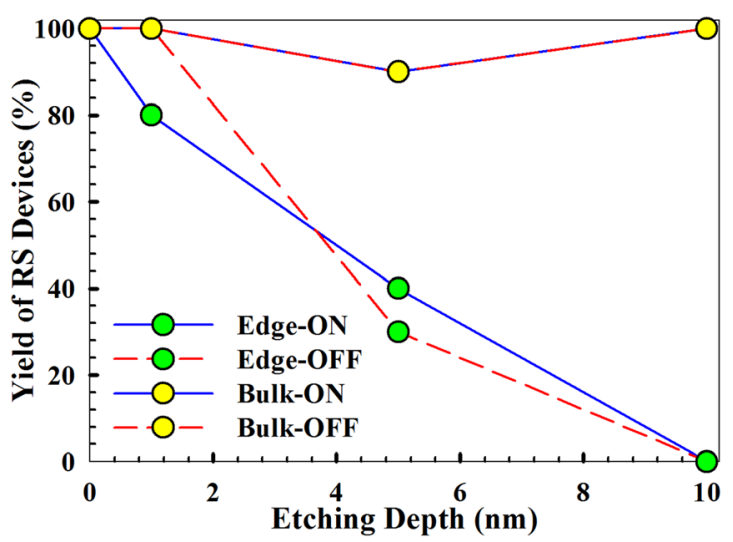

FIG. 3. Yield (percentage) of devices remaining intact after dilute HF etch to various depths. necessary for $\mathrm{SiO}_{\mathrm{x}}$-based devices to electroform and switch, ${ }^{14,15}$ these results demonstrate that this is not the case. The reason why encapsulated edge devices fail to switch in air may be related to the detailed structure of the edge. When the isotropic $\mathrm{BOE}$ forms the $\mathrm{SiO}_{\mathrm{x}}$ edge, it creates a cavity underneath the top electrode that poses a problem for encapsulation. The inset of Figure 2(b) shows a cross-section scanning electron microscope (SEM) image of an edge device with SU-8 encapsulation, where the cavity is clearly visible. As the encapsulation process was done in cleanroom air, it is expected that oxygen was trapped within the cavity, and this may cause device oxidization and switching failure.

Figure 4 shows both the measurement results and the cross-sectional SEM image of the bulk device with SU-8 encapsulation. The bulk device switches from HRS to LRS in the range from $2.5 \mathrm{~V}$ to $3.5 \mathrm{~V}$; and from LRS to HRS in the range from $4.5 \mathrm{~V}$ to $5.5 \mathrm{~V}$. Interestingly, the device $\mathrm{On} /$ Off ratio in Figure 4 is above $10,{ }^{6}$ which is even larger than the On/Off ratio measured in vacuum (Figure 1(b)). ${ }^{11}$ The resistive switching of bulk $\mathrm{SiO}_{2}$ devices with SU-8 encapsulation in air ambient demonstrates that reversible switching does not require vacuum conditions or a non-oxidizing ambient. This is consistent with the hypothesis that state switching corresponds to a transformation between two different hydrogen-complexed defects, namely, the hydrogen bridge

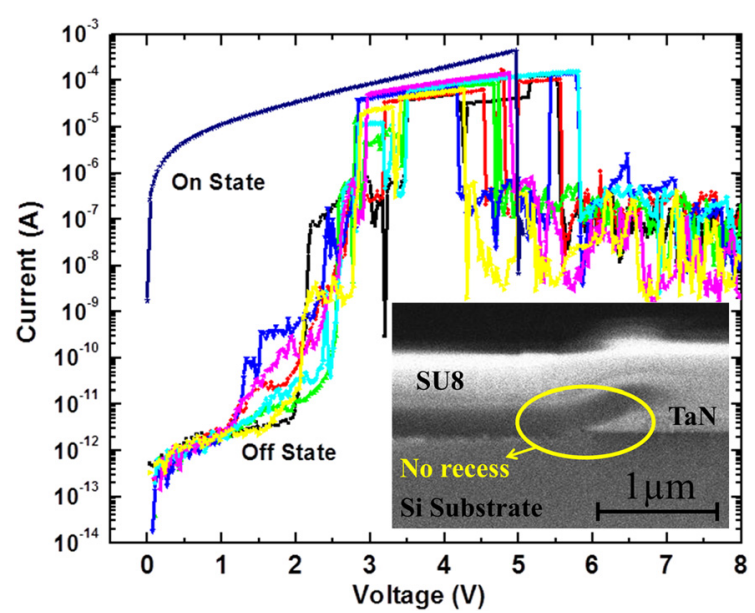

FIG. 4. Resistive switching of encapsulated bulk device measured in air ambient. The inset shows the cross-section SEM image of the bulk device with SU-8 encapsulation. 


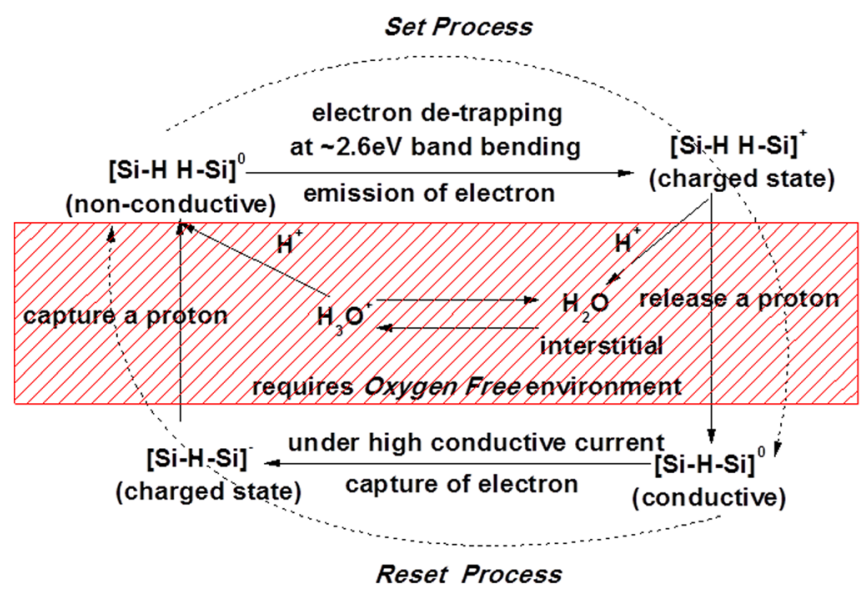

FIG. 5. Chart showing hypothesized hydrogen-complexed defect transitions during reversible switching. Non-conductive defect $[\mathrm{Si}-\mathrm{H} \mathrm{H}-\mathrm{Si}]^{0}$ (top left). Charged state $[\mathrm{Si}-\mathrm{H} \mathrm{H}-\mathrm{Si}]^{+}$due to band bending (top left). Conductive defect $[\mathrm{Si}-\mathrm{H}-\mathrm{Si}]^{0}$ (bottom right). Charged state $[\mathrm{Si}-\mathrm{H}-\mathrm{Si}]^{-}$(bottom left) due to electron capture during high current. Interchangeable defects $\mathrm{H}_{2} \mathrm{O}$ and $\mathrm{H}_{3} \mathrm{O}^{+}$(middle). Reactions in the red-shaded area require oxygen-free environment.

( $\mathrm{Si}-\mathrm{H}-\mathrm{Si})$ and hydrogen doublet $(\mathrm{Si}-\mathrm{H} \mathrm{H}-\mathrm{Si}) .{ }^{16-18} \mathrm{~A}$ chart showing device state switching and the participating defects is shown in Figure 5. The set process is initiated by band bending of $2.6 \mathrm{eV}$ which charges the $\mathrm{Si}-\mathrm{H} \mathrm{H}$-Si defect positive and releases a proton, followed by proton uptake by $\mathrm{H}_{2} \mathrm{O}$ to form a potentially stable dipole complex, Si-H$\mathrm{Si}^{-}+\mathrm{H}_{3} \mathrm{O}^{+}$, where the $\mathrm{Si}-\mathrm{H}-\mathrm{Si}$ defect supports high current flow in the conductive state. ${ }^{19,20}$ The reset process is modeled as being initiated by electron injection into both $\mathrm{Si}-\mathrm{H}-\mathrm{Si}$ and $\mathrm{H}_{3} \mathrm{O}^{+}$, charging the $\mathrm{Si}-\mathrm{H}-\mathrm{Si}$ defect negative and leading to proton release from $\mathrm{H}_{3} \mathrm{O}^{+}$and reaction with $\mathrm{Si}-\mathrm{H}^{-}-\mathrm{Si}$ to form $\mathrm{H}_{2} \mathrm{O}$ (interstitial) $+\mathrm{Si}-\mathrm{H} \mathrm{H}-\mathrm{Si}$ in the non-conductive state. ${ }^{17}$ This electrochemical model provides a simple, yet comprehensive explanation for the unique unipolar operating characteristics of the $\mathrm{SiO}_{\mathrm{x}}$ RRAM device. It is obvious that both transformations require an environment free of unbonded oxygen ions or atoms, shown as the shaded area in Figure 5, to prevent formation of $\mathrm{Si}-\mathrm{O}$ bonds during state switching when H-passivation of $\mathrm{Si}$ is temporarily lost. This model also helps to explain the destructive role of oxygen during the resistive switching process. The fact that encapsulated bulk devices can switch state in $1 \mathrm{~atm}$ of air demonstrates that SU-8 epoxy provides an effective hermetic seal to prevent oxygen in the air from reaching the $\mathrm{SiO}_{\mathrm{x}}$ layer, thus allowing the defect transformations to take place. On the other hand, it is believed that air trapped within the cavity of encapsulated edge devices prevents RRAM switching, where oxygen in the cavity can react with the defects responsible for resistive state switching.

In conclusion, $\mathrm{SiO}_{\mathrm{x}}$ RRAM bulk and edge devices have been electroformed and tested in vacuum. Electrical test in oxygen ambient shows that un-encapsulated edge devices lose resistive switching capability and memory characteristics, most likely due to oxidation of the Si-rich filament. With SU-8 epoxy encapsulation, the bulk device achieves stable state switching in air ambient. The SU-8 epoxy is believed to hermetically seal the device to prevent oxygen from permeating into the $\mathrm{SiO}_{\mathrm{x}}$ layer, thus enabling reversible switching in air. This work illustrates that, with proper structure and encapsulation, the $\mathrm{SiO}_{\mathrm{x}} \mathrm{RRAM}$ can be operated in air ambient, which is a critically important step for integration with CMOS technology and use in advanced nonvolatile memory applications.

This material is based upon work supported by the National Science Foundation under Grant No. IIP-1127537.

${ }^{1}$ M. Ziegler and M. Stan, IEEE Trans. Nanotechnol. 2, 217 (2003).

${ }^{2}$ E. Linn, R. Rosezin, C. Kugeler, and R. Waser, Nat. Mater. 9, 403 (2010).

${ }^{3}$ F. Zhou, Y.-F. Chang, K. Byun, B. Fowler, and J. C. Lee, Appl. Phys. Lett. 105, 133501 (2014).

${ }^{4}$ Y. Wang, B. Fowler, Y. Chen, and J. Lee, Appl. Phys. Lett. 100, 083502 (2012).

${ }^{5}$ Y. Chang, Y. Chen, F. Xue, and J. Lee, Appl. Phys. Lett. 101, 052111 (2012).

${ }^{6}$ J. Yao, Z. Sun, L. Zhong, D. Natelson, and J. Tour, Nano Lett. 10, 4105 (2010).

${ }^{7}$ Y. Wang, B. Fowler, Y. Chen, and J. Lee, Appl. Phys. Lett. 101, 183505 (2012).

${ }^{8}$ Y. T. Chen, B. Fowler, Y. Wang, and J. C. Lee, IEEE Electron Device Lett. 33, 1702 (2012).

${ }^{9}$ A. Mehonic, S. Cueff, M. Wojdak, and O. Jambois, J. Appl. Phys. 111, 074507 (2012).

${ }^{10}$ G. Murillo, Z. J. Davis, S. Keller, and N. Barniol, Microelectron. Eng. 87, 1173 (2010).

${ }^{11}$ Y. Chang, L. Ji, B. Fowler, and J. Lee, Appl. Phys. Lett. 103, 033521 (2013).

${ }^{12}$ Y. Chang, B. Fowler, and J. C. Lee, J. Appl. Phys. 116, 043708 (2014).

${ }^{13}$ Y. Chang, L. Ji, Y. Wang, F. Zhou, B. Fowler, and J. C. Lee, Appl. Phys. Lett. 103(19), 193508 (2013).

${ }^{14}$ Y.-F. Chang, B. Fowler, Y.-C. Chen, Y.-T. Chen, Y. Wang, F. Xue, F. Zhou, and J. C. Lee, J. Appl. Phys. 116, 043709 (2014).

${ }^{15}$ Y. Chang, P. Chen, B. Fowler, and J. Lee, J. Appl. Phys. 112, 123702 (2012).

${ }^{16}$ J. Robertson, J. Phys. C: Solid State Phys. 17, L221-L225 (1984).

${ }^{17}$ P. E. Blochl, Phys. Rev. B 62, 6158, (2000).

${ }^{18}$ S. N. Rashkeev, M. Di Ventra, and S. T. Pantelides, Appl. Phys. Lett. 78, 1571 (2001).

${ }^{19}$ J. Suñé and E. Wu, Int. Electron Devices Meet. 2005, 388.

${ }^{20}$ P. E. Blöchl and J. H. Stathis, Phys. Rev. Lett. 83, 372 (1999).

${ }^{21}$ S. W. King, J. Vac. Sci. Technol., A 29, 041501 (2011).

${ }^{22}$ S. W. King, D. Jacob, D. Vanleuven, B. Colvin, J. Kelly, M. French, J. Bielefeld, D. Dutta, M. Liu, and D. Gidley, ECS J. Solid State Sci. Technol. 1, N115 (2012). 